\title{
Translation Inhibition of Capped and Uncapped Viral RNAs Mediated by Ribosome-Inactivating Proteins
}

\author{
Jorge M. Vivanco and Nilgun E. Tumer
}

Department of Plant Biology and Pathology and The Biotechnology Center for Agriculture and the Environment, Rutgers University, New Brunswick 08901.

Current address of J. M. Vivanco: Department of Horticulture and Landscape Architecture, Colorado State University, Fort Collins 80523. Accepted for publication 13 November 2002.

\begin{abstract}
Vivanco, J. M., and Tumer, N. E. 2003. Translation inhibition of capped and uncapped viral RNAs mediated by ribosome-inactivating proteins. Phytopathology 93:588-595.

Ribosome-inactivating proteins (RIPs) are N-glycosidases that remove specific purine residues from the sarcin/ricin (S/R) loop of the large rRNA and arrest protein synthesis at the translocation step. In addition to their enzymatic activity, RIPs have been reputed to be potent antiviral agents against many plant, animal, and human viruses. We recently showed that pokeweed antiviral protein (PAP), an RIP from pokeweed, inhibits translation in cell extracts by binding to the cap structure of eukaryotic mRNA and viral RNAs and depurinating these RNAs at multiple sites downstream of the cap structure. In this study, we examined the activity of three different RIPs against capped and uncapped viral RNAs. PAP, Mirabilis expansa RIP (ME1), and the Saponaria officinalis RIP (saporin) depurinated the capped Tobacco mosaic virus and Brome

mosaic virus RNAs, but did not depurinate the uncapped luciferase RNA, indicating that other type I RIPs besides PAP can distinguish between capped and uncapped RNAs. We did not detect depurination of Alfalfa mosaic virus (AMV) RNAs at multiple sites by PAP or ME1. Because AMV RNAs are capped, these results indicate that recognition of the cap structure alone is not sufficient for depurination of the RNA at multiple sites throughout its sequence. Furthermore, PAP did not cause detectable depurination of uncapped RNAs from Tomato bushy stunt virus (TBSV), Satellite panicum mosaic virus (SPMV), and uncapped RNA containing poliovirus internal ribosome entry site (IRES). However, in vitro translation experiments showed that PAP inhibited translation of AMV, TBSV, SPMV RNAs, and poliovirus IRES dependent translation. These results demonstrate that PAP does not depurinate every capped RNA and that PAP can inhibit translation of uncapped viral RNAs in vitro without causing detectable depurination at multiple sites. Thus, the cap structure is not the only determinant for inhibition of translation by PAP.
\end{abstract}

Ribosome-inactivating proteins (RIPs) are widely distributed plant enzymes that inhibit protein synthesis by virtue of their $\mathrm{N}$ glycosidase activity, selectively cleaving adenine and guanine residues within the highly conserved sarcin/ricin (S/R) loop of the $28 \mathrm{~S}$ rRNA $(10,18)$. This cleavage prevents the binding of the EF2/GTP complex, with the subsequent arrest of protein synthesis at the elongation step $(24,28)$. Besides their activity on rRNA, certain RIPs display a variety of antimicrobial activities in vitro, such as antifungal, antibacterial $(38,43)$, and broad-spectrum antiviral activity against both plant and animal viruses, including the human immunodeficiency virus, HIV-1 $(27,40)$.

The classical view of the antiviral role of RIPs in plants is based mostly on their enzymatic activity and compartmentalization $(8,31,32)$. Because RIPs may potentially inactivate ribosomes in the cells in which they are synthesized, they are sequestered in vacuoles, protein bodies, or cell walls $(8,31)$. Based on their enzymatic activity and subcellular location, it has been suggested that RIPs may be "suicidal agents" released from cell walls or vacuoles after a plant cell is infected by a virus $(3,31)$. It is thought that when plants are wounded during viral infection, RIPs are released from their cellular compartments to depurinate the $28 \mathrm{~S}$ rRNA. This would prevent viral replication at an early stage by inactivating the cell protein synthesis machinery and leading to autonomous cell death $(27,40)$.

Our group and others have reported that RIPs may use other nucleic acids as substrates, including poly(A) RNA, herring sperm

Corresponding author: N. E. Tumer; E-mail address: tumer@aesop.rutgers.edu

Publication no. P-2003-0221-02R

(C) 2003 The American Phytopathological Society
DNA, and capped RNAs, indicating that RIPs may depurinate substrates other than rRNA $(2,18,33)$. Saporin from Saponaria officinalis depurinates a variety of polynucleotides (2). To date, more than 50 RIPs have been shown to be capable of depurinating DNA, RNA, and poly(A) RNA (3). Some RIPs were reported to hydrolyze single-stranded and double-stranded DNA $(21,22,26$, 39). We have recently shown that pokeweed antiviral protein (PAP) mutants inhibit translation of capped, but not uncapped, RNAs in cell extracts without depurinating the S/R loop (18). Translation of Brome mosaic virus (BMV), a capped RNA-containing virus, was also inhibited by PAP, and its effect was overcome by increasing concentrations of the cap analog $\mathrm{m}^{7} \mathrm{GpppG}$ (18). Analysis of the interaction between the cap structure and PAP indicated that PAP binds to the $\mathrm{m}^{7} \mathrm{GpppG}$ cap structure but does not remove the cap (16). PAP depurinates the luciferase RNA downstream of the cap at multiple sites throughout the mRNA sequence (16). These results demonstrate that PAP could inhibit translation by targeting the mRNA rather than the rRNA $(16,18)$.

In this study, we examined the activity of three different RIPs, PAP, Mirabilis expansa (ME1), and saporin, against capped and uncapped viral RNAs. We present evidence that single chain (type I) RIPs vary in their activity against different capped viral RNAs and they do not depurinate every capped RNA, indicating that they display selectivity. Although PAP did not cause detectable depurination of uncapped viral RNAs, it inhibited their translation, indicating that the cap structure is not the only determinant for inhibition of translation by PAP.

\section{MATERIALS AND METHODS}

Purification of RIPs. Purified saporin was purchased from Sigma Chemical (St. Louis, MO). Purification of PAP from poke- 
weed leaves was performed as described by Wang and Tumer (39). Ammonium sulfate precipitation was used to sediment PAP protein from homogenized leaves that were dialyzed to remove excess salt. Negatively charged proteins were excluded from the mix by passing the dialysate through Q15 anion exchange filters connected to a fast protein liquid chromatography system (BioRad Biologic System; Bio-Rad, Hercules, CA). Positively charged proteins were collected in the flow-through and loaded in a S2 cation exchange column (Bio-Rad). PAP proteins were eluted from the column using 0 to $30 \% \mathrm{NaCl}$ gradient. Fractions were assayed by immunoblot analysis and those containing PAP were pooled and concentrated by ultrafiltration with a Microcon 10-kDa cut-off filter (Amicon, Millipore, Bedford, MA). ME1 was purified following the method described by Vivanco et al. (38). The protein solution from roots was concentrated to $1 \mathrm{mg} / \mathrm{ml}$ using a Stirred Cell 8050 (Amicon) with a YM10 membrane. Subsequently, cation exchange separation was performed using POROS perfusion chromatography (PE Applied Biosystems, Foster City, CA). A column (4.6 by $100 \mathrm{~mm} ; 1.66 \mathrm{ml}$ volume) was packed with POROS $20 \mathrm{HS}$ cation-exchange perfusion media (PE Applied Biosystems) and used in conjunction with a highperformance liquid chromatography system (Model 600E; Waters, Milford, MA) equipped with a photodiode array detector (Model 990; Waters). Equilibration, loading, and washing were carried out in $25 \mathrm{mM} \mathrm{N}$-2-hydroxyethylpiperazine- $N$ '-2-ethanesulfonic acid (pH 8.0) containing $50 \mathrm{mM} \mathrm{NaCl}$. The target proteins were eluted with a linear gradient of 30 column volumes (approximately $50 \mathrm{ml}$ ) from 50 to $200 \mathrm{mM} \mathrm{NaCl}$ at a flow rate of $5 \mathrm{ml} / \mathrm{min}$.

RNA templates. BMV RNAs were purchased from Promega (Madison, WI). Tobacco mosaic virus (TMV) and Alfalfa mosaic virus (AMV) RNAs were extracted from purified viral preparations. Satellite panicum mosaic virus (SPMV) and Tomato bushy stunt virus (TBSV) RNAs were either extracted from purified virions or synthesized as run-off transcripts from linearized cDNA templates (provided by K. Scholthof and H. Scholthof, Texas A\&M University). Poliovirus RNAs were transcribed in vitro using the pcDNA3 (LUC-polioIRES-CAT) plasmid, which contains the luciferase open reading frame, poliovirus internal ribosome entry site (IRES), and chloramphenicol acetyl transferase (CAT) open reading frame downstream of the $\mathrm{T} 7$ promoter in pcDNA3 (Invitrogen) (provided by N. Sonenberg, McGill University). Uncapped luciferase mRNA was synthesized as run-off transcripts of a T7 polymerase reaction using linearized luciferase DNA as template (Promega).

Electrophoresis analysis. Sodium dodecyl sulfate-polyacrylamide gel electrophoresis (SDS-PAGE) was performed with 13.5 or $15 \%$ acrylamide discontinuous gels (20) using a Mini-Protean II electrophoresis cell (Bio-Rad) following the manufacturer's instructions. A low-molecular mass (14 to $66 \mathrm{kDa})$ protein marker kit (Bio-Rad) was used to determine approximate protein sizes. Proteins were visualized with Coomassie brilliant blue G-250 (Calbiochem, La Jolla, CA) or silver staining (Bio-Rad).

RNA depurination assay. The assay was conducted according to Tumer et al. (36). Briefly, viral or luciferase RNA (500 ng) was resuspended in RIP buffer $(167 \mathrm{mM} \mathrm{KCl}, 100 \mathrm{mM}$ Tris- $\mathrm{HCl}, \mathrm{pH}$ 7.2, and $100 \mathrm{mM} \mathrm{MgCl}_{2}$ ) and incubated with different concentrations of PAP, ME, and saporin, respectively, at $30^{\circ} \mathrm{C}$ for $30 \mathrm{~min}$ in a total volume of $100 \mu \mathrm{l}$. RNA incubated in the absence of RIPs served as a negative control. Following incubation, the RIPs were removed from the mixture by phenol/chloroform extraction and the RNA was divided in half. Half of the RNA extracted was incubated on ice for $30 \mathrm{~min}$ with $1 \mathrm{M}$ aniline acetate $(\mathrm{pH} 4.5)$ and precipitated with ethanol. Both aniline-treated and -untreated RNAs were electrophoresed in a $7 \mathrm{M}$ urea/6\% polyacrylamide gel and stained with ethidium bromide.

In vitro translation. Viral RNA (500 ng) was incubated with different concentrations of RIPs. Viral RNA was removed from the RIP incubation mixture by phenol/chloroform extraction and was precipitated in ethanol at $-80^{\circ} \mathrm{C}$. The RIP-treated viral RNA was resuspended in diethylprocarbonate water and added to a rabbit reticulocyte lysate in vitro translation system (Promega). The translation mixture $(25 \mu \mathrm{l})$ was incubated at $30^{\circ} \mathrm{C}$ for $1.5 \mathrm{~h}$; total protein products were trichloroacetic acid (TCA)-precipitated and the level of translation was measured by $\left[{ }^{35} \mathrm{~S}\right]$-methionine incorporation. In vitro-translated proteins were visualized by autoradiography of SDS-PAGE gels. Briefly, $5 \mu$ of the total $25-\mu 1$ translation mixture was separated by $12 \%$ SDS-PAGE. The gel was dried and exposed to X-ray film. In vitro translation products were quantified with a scintillation counter.

\section{RESULTS}

Effect of ME and saporin on BMV RNAs. We have previously shown that incubation of BMV RNAs with PAP leads to inhibition of translation by a mechanism other than rRNA depurination (18). PAP binds to the cap structure and depurinates the capped RNA template (16). To determine if other RIPs have similar activity on capped RNAs, we incubated ME1 and saporin with BMV RNAs. ME1 is an RIP from Mirabilis expansa (ME), which had previously been purified and partially characterized (38). We used highly purified fractions of PAP, ME1, and saporin that were homogenous, as determined by SDS-PAGE analysis and silver staining as previously described $(38,39)$. The effect of RIPs on capped BMV RNAs was determined by incubating the RIPtreated RNA with acidic aniline. The phosphodiester bond at the $3^{\prime}$ end of the depurinated site in the RNA is susceptible to hydrolysis by $\beta$-elimination in the presence of aniline (36). Treatment of depurinated RNA with acidic aniline causes chain cleavage at the depurination sites $(17,36)$. Therefore, complete degradation of the RNA after aniline treatment will occur only if the RNA has been depurinated at multiple sites. Following incubation of PAP, ME1 (ME), and saporin with BMV RNAs, viral RNA was extracted and one-half of the treated RNA was incubated with aniline. As shown in Figure 1A, $1 \mathrm{ng}$ of each RIP was sufficient to depurinate BMV RNAs at multiple sites throughout their sequence, as indicated by complete degradation of the RNAs after treatment with aniline. At higher RIP concentrations, degradation of RNA was observed in the absence of aniline, as previously shown with PAP-treated BMV and capped luciferase RNAs (18). To compare the cap specificity of ME1 and saporin with PAP (18), uncapped luciferase transcripts were incubated with ME1 and saporin. ME1 and saporin did not depurinate the uncapped luciferase transcripts after treatment with aniline (Fig. 1B). As previously shown, up to $100 \mathrm{ng}$ of PAP did not depurinate the uncapped luciferase transcripts (18). In contrast, capped luciferase transcripts previously incubated with ME1 and saporin exhibited RNA depurination after treatment with aniline as previously observed with PAP (18). These results demonstrate that the activity of ME1 and saporin on BMV RNAs is comparable to the previously reported activity of PAP on BMV RNAs (18).

Effect of PAP, ME, and saporin on TMV RNA. To determine if RIP depurination of viral RNA is mediated by cap recognition, we isolated TMV RNA from a highly purified virus preparation. TMV RNA is capped at its $5^{\prime}$ end and contains a histidineaccepting tRNA-like structure at its $3^{\prime}$ end. TMV RNA was incubated with PAP, ME1, and saporin, respectively. Subsequently, viral RNA was extracted, treated with aniline, and separated by electrophoresis as described previously. As shown in Figure 2, the RIPs recognized and depurinated TMV RNA. However, enzymatic activity was evident at higher concentrations of RIPs and was not observed at low RIP concentrations. Depurination of TMV RNA was evident only after aniline treatment of RNA incubated with $100 \mathrm{ng}$ of PAP and ME1 (Fig. 2). Even after incubation with $100 \mathrm{ng}$ of PAP and ME1, TMV RNA was not completely degraded when treated with aniline. Total degradation of TMV RNA was observed with $100 \mathrm{ng}$ of saporin even without aniline 
treatment. Because TMV RNA treated with $1 \mathrm{ng}$ of saporin was not completely degraded after aniline treatment, depurination of the TMV RNA occurs after treatment with higher amounts of saporin. These results indicate that PAP, ME1, and saporin recognize and depurinate the capped TMV RNA. Higher concentrations of all three RIPs are needed for depurination of TMV RNA than for BMV RNAs, demonstrating that although RIPs may recognize the cap structure of BMV and TMV RNAs, they differ in activity toward the two different capped viral RNAs.

Effect of PAP, ME, and saporin on AMV RNAs. Based on the distinct depurination results using BMV and TMV RNAs, and considering the fact that BMV is composed of three genomic capped RNAs, whereas TMV has only one capped RNA, we decided to test the activity of the different RIPs on AMV, a multipartite RNA virus. AMV is composed of three genomic and one subgenomic capped RNAs. The genomic RNAs of AMV contain hairpin structures downstream of the cap, which have been extensively analyzed (1,35). Furthermore, unlike BMV and TMV RNAs, AMV RNAs do not contain a tRNA-like structure at their 3 ' ends. The enzymatic activities of PAP, ME1, and saporin were tested against AMV RNAs. Surprisingly, as shown in Figure 3, depurination at multiple sites was not observed even at higher concentrations of PAP and ME1. RNA degradation was only ob-

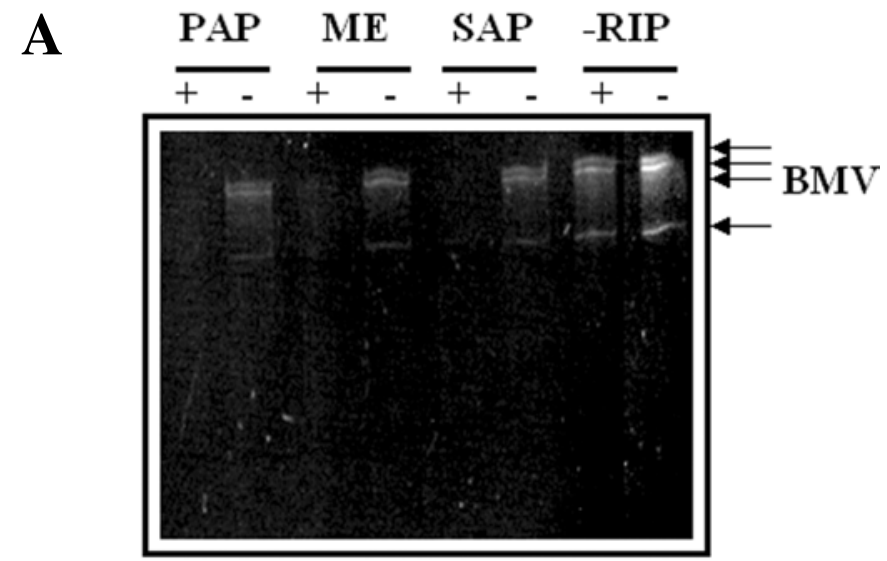

B

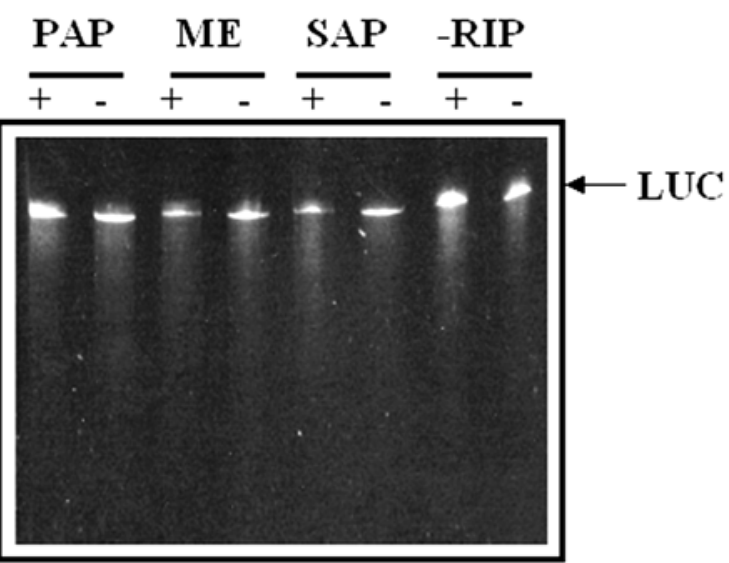

Fig. 1. In vitro depurination of capped and uncapped RNAs by pokeweed antiviral protein (PAP), ME1 from Mirabilis expansa (ME), and saporin from Saponaria officinalis (SAP). Brome mosaic virus (BMV) A, capped RNAs (500 ng) or B, uncapped luciferase (LUC) transcripts (500 ng) were incubated with $1 \mathrm{ng}$ of PAP, ME, and saporin, respectively. RNAs were incubated with the ribosome-inactivating proteins (RIPs) as described in the text. RNA was extracted, treated with aniline, separated on a $7 \mathrm{M}$ urea/6\% polyacrylamide gel, and stained with ethidium bromide. The presence $(+)$ or absence (-) of aniline is denoted. Arrows indicate A, the four full-length BMV RNAs (from top to bottom) of 3.2-, 2.8-, 2.1-, and 0.8-kb and $\mathbf{B}$, the $1-\mathrm{kb}$ uncapped luciferase (LUC) transcript. -RIP refers to RNA controls incubated in buffer. served in samples previously incubated with $100 \mathrm{ng}$ of saporin and treated with aniline. AMV RNAs did not separate well on the $7 \mathrm{M}$ urea/6\% acrylamide gel because these gels were run only long enough to detect small RNA fragments released upon aniline treatment. These results provide further evidence that cap recognition is important but not sufficient for RIP depurination of capped RNA templates.

PAP inhibits translation of AMV RNAs. To examine the efficiency of translation of AMV RNAs previously treated with RIPs, RIP-treated AMV RNAs were translated in a rabbit reticulocyte lysate. AMV RNAs were incubated with PAP or ME1 prior to translation. PAP and ME1 were then removed from the incubation mixture by phenol/chloroform extraction to guarantee that if reduction in translation was observed it was due to the RIP-treated RNAs rather than an effect of the RIPs on the reticulocyte lysate. Translation was measured by $\left[{ }^{35} \mathrm{~S}\right]$-methionine incorporation into protein products and analyzed by autoradiography of SDS-PAGE gels and scintillation counter quantification. Because RIP treatment of AMV RNAs did not show evidence of depurination, we expected no inhibition of translation of these RIP-treated RNAs. However, as shown in Figure 4A, translation products (126, 90, 32 , and $24 \mathrm{kDa}$ ) were significantly reduced in AMV RNAs previously treated with $100 \mathrm{pg}$ and $5 \mathrm{ng}$ of PAP. In contrast, the
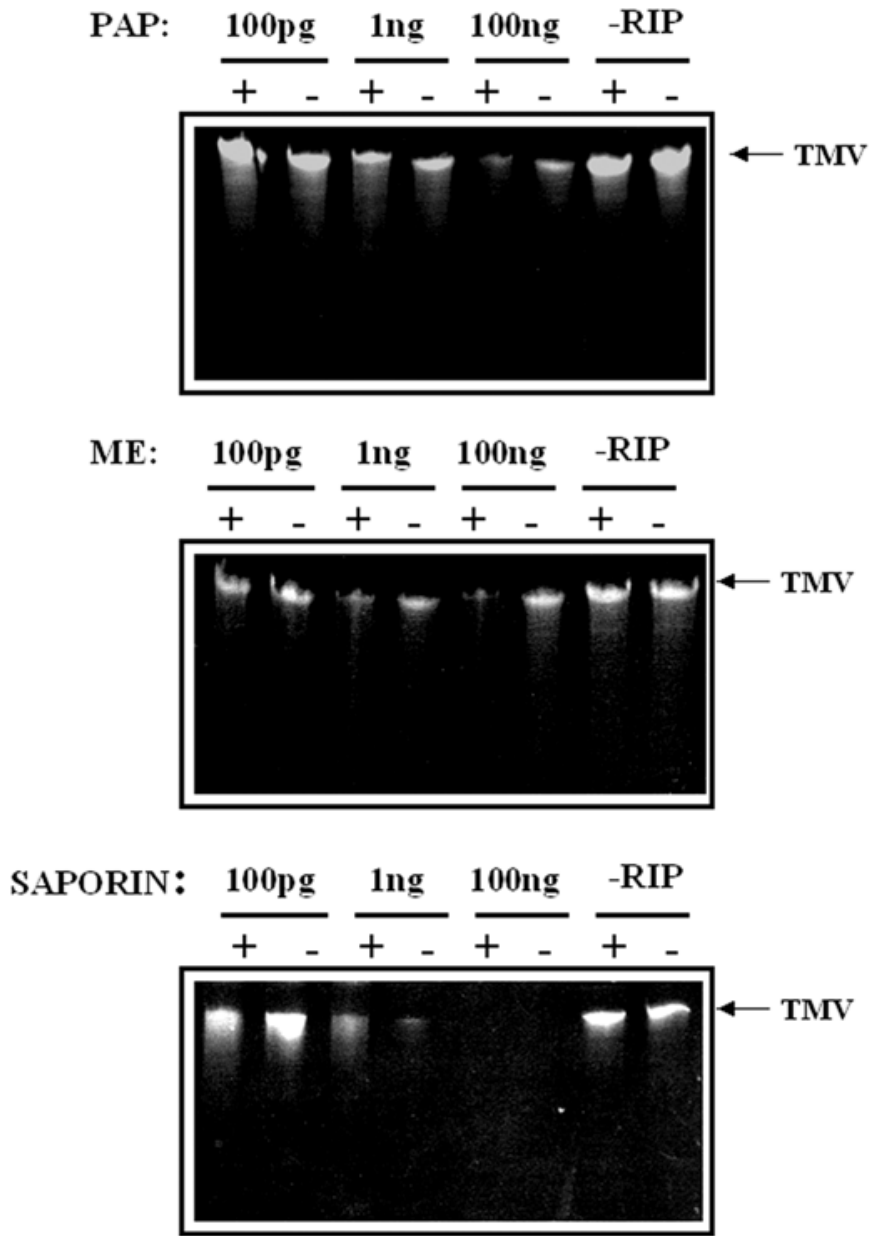

Fig. 2. In vitro depurination of capped Tobacco mosaic virus (TMV) RNA (500 ng) by pokeweed antiviral protein (PAP), ME1 from Mirabilis expansa (ME), and saporin from Saponaria officinalis (saporin), respectively. RNAs were incubated with the ribosome-inactivating proteins (RIPs) as described in the text. RNA was extracted, treated with aniline, separated on a $7 \mathrm{M}$ urea/ $6 \%$ polyacrylamide gel, and stained with ethidium bromide. The presence $(+)$ or absence $(-)$ of aniline is denoted. TMV RNA was underloaded in the lane that shows treatment with $1 \mathrm{ng}$ of saporin in the absence of aniline (-). Arrows indicate 6.3-kb TMV RNA. -RIP refers to RNA controls incubated with buffer. 
amount of translation products from the uncapped luciferase transcripts did not decrease after treatment with $100 \mathrm{pg}$ of PAP and decreased only slightly after treatment with $5 \mathrm{ng}$ of PAP (Fig. 4A). ME1-treated AMV RNAs did not show a significant decrease in translation efficiency compared with untreated AMV RNA control. As expected, uncapped luciferase transcripts previously treated with ME1 did not show inhibition of translation (Fig. 4B). To demonstrate that the reduction in translation of PAP-treated AMV RNAs was not due to contaminating RNase activity present in the protein preparations, AMV RNAs incubated with PAP were separated on a $7 \mathrm{M}$ urea/6\% polyacrylamide gel. As seen in Figure 4C, AMV RNAs previously treated with PAP, and used in the rabbit reticulocyte lysate translation assay, did not show any evidence of degradation. Similarly, uncapped luciferase transcripts treated with PAP were not degraded (Fig. 4C).

The samples used for autoradiography were TCA-precipitated and quantified in a scintillation counter. As shown in Figure 5A, in vitro translation of AMV RNAs previously incubated with PAP resulted in a significant decrease in overall incorporation of $\left[{ }^{35} \mathrm{~S}\right]-$ methionine into TCA-precipitable material compared with AMV RNAs translated without PAP pretreatment. PAP at 100 pg inhibited the level of translation by $88 \%$ compared with AMV RNAs treated with buffer. At $5 \mathrm{ng}$, PAP showed $94 \%$ inhibition of AMV translation. PAP did not inhibit overall translation of un-
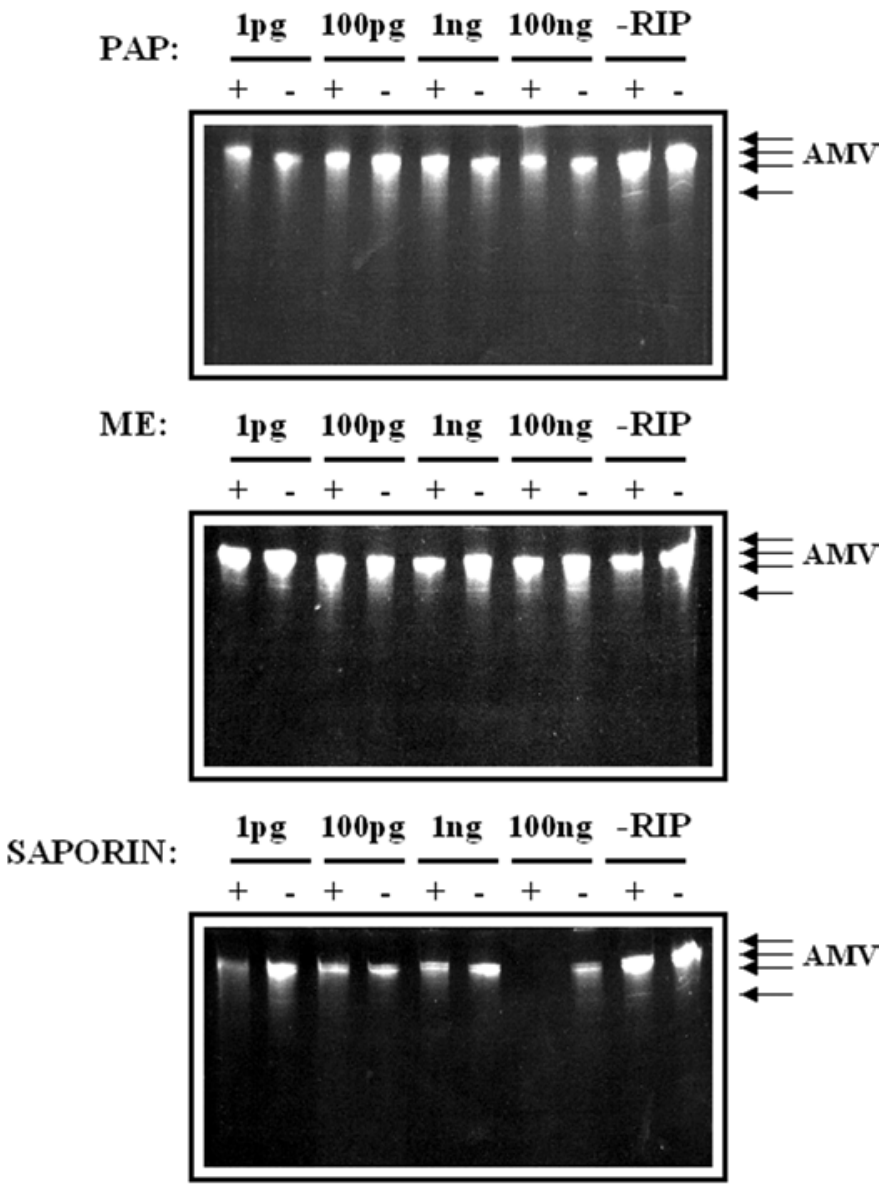

Fig. 3. In vitro depurination of capped Alfalfa mosaic virus (AMV) RNAs by pokeweed antiviral protein (PAP), ME1 from Mirabilis expansa (ME), and saporin from Saponaria officinalis (saporin), respectively. Capped AMV RNAs (500 ng) were incubated with the ribosome-inactivating proteins (RIPs) as described in the text. RNA was extracted, treated with aniline, separated on a $7 \mathrm{M}$ urea/6\% polyacrylamide gel, and stained with ethidium bromide. The presence $(+)$ or absence $(-)$ of aniline is denoted. In each panel, from top down, arrows indicate the 3.6-, 2.5-, and 2.2-kb AMV RNAs 1,2 , and 3, and lower arrows indicate the 0.6-kb AMV RNA 4. -RIP refers to RNA controls incubated with buffer. capped luciferase RNA. As shown in Figure 5B, ME1 did not show a significant inhibition of the overall levels of translation for either AMV RNAs or uncapped luciferase transcripts. PAP-mediated inhibition of translation of AMV RNAs without depurination at multiple sites indicates that recognition of the cap structure alone is not sufficient for depurination of the RNA at multiple sites. In contrast to PAP, ME1 did not inhibit translation of AMV RNAs, providing further evidence that RIPs differ in their specificity toward different capped viral RNA templates.

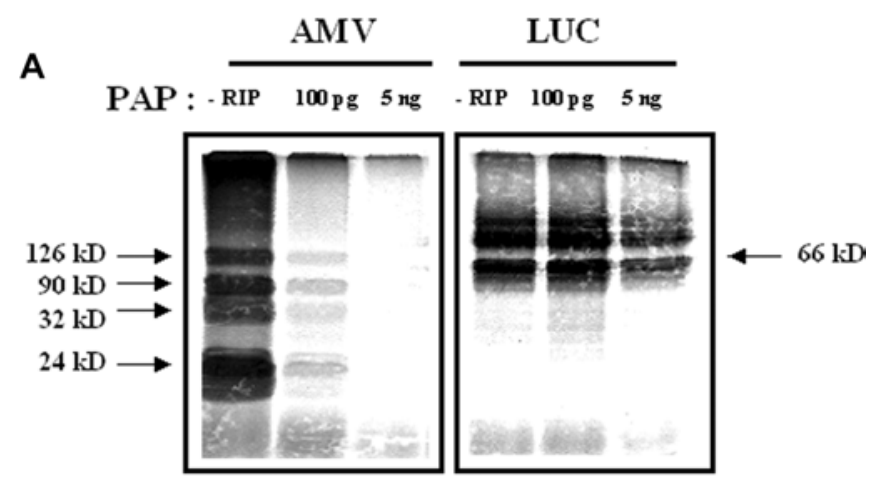

B

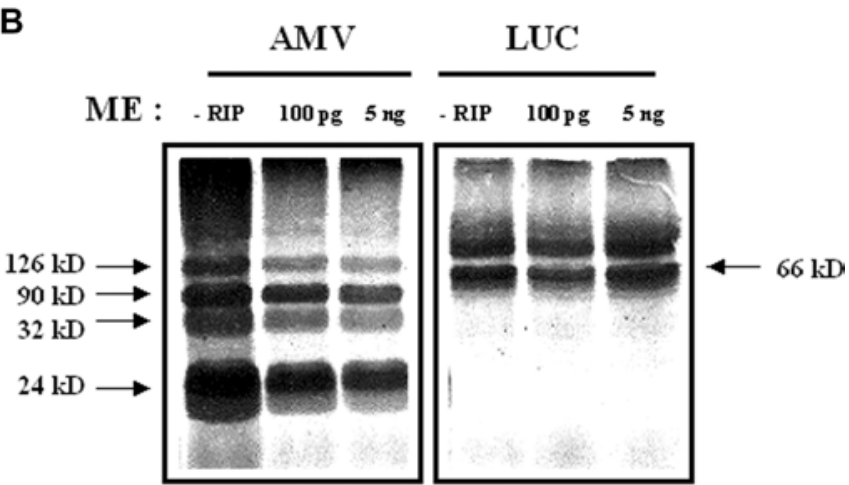

C

PAP : - RIP 100pg $5 \mathrm{ng}$ - RIP $100 \mathrm{pg} 5 \mathrm{ng}$

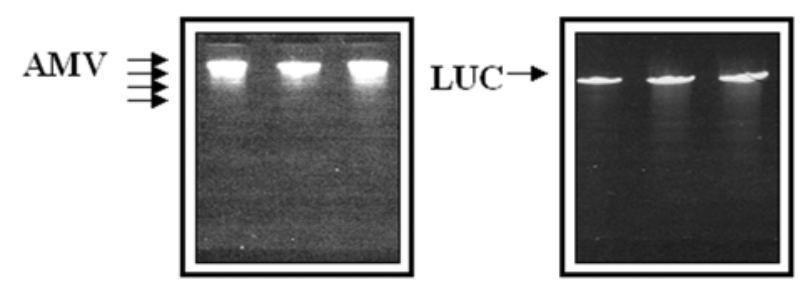

Fig. 4. Autoradiograms illustrating the translation inhibition of capped Alfalfa mosaic virus (AMV) RNAs and uncapped luciferase (LUC) transcripts by pokeweed antiviral protein (PAP) and ME1 from Mirabilis expansa (ME). AMV RNAs (500 ng) and capped luciferase (LUC) transcripts were previously incubated with $100 \mathrm{pg}$ and $5 \mathrm{ng}$ of A, PAP and $\mathbf{B}$, $\mathrm{ME}$ and translated in a rabbit reticulocyte translation system. $\mathbf{A}$ and $\mathbf{B}$, Five microliters of the total $25-\mu$ incubation mixture was separated on a $7 \mathrm{M}$ urea/6\% polyacrylamide gel and analyzed by autoradiography. Arrows indicate the proteins translated by AMV RNAs. C, AMV RNAs and uncapped luciferase (LUC) transcripts treated with $100 \mathrm{pg}$ and $5 \mathrm{ng}$ of PAP as indicated in $\mathbf{A}$ and $\mathbf{B}$. RNAs were incubated with the ribosome-inactivating proteins (RIPs) as described in the text. RNA was extracted and separated on a $7 \mathrm{M}$ urea/6\% polyacrylamide gel and stained with ethidium bromide. In the AMV panel, upper arrow indicates the 3.6-, 2.5-, and 2.2-kb AMV RNAs 1, 2 , and 3, and lower arrow indicates the 0.6-kb AMV RNA 4. In the luciferase panel, the arrow indicates the 1-kb uncapped luciferase transcript. -RIP refers to RNA controls incubated with buffer. RNA controls (-) incubated with buffer are denoted. 
PAP inhibits translation from poliovirus IRES, TBSV, and SPMV RNAs without depurination. Because RNA depurination or translation inhibition was not observed when uncapped luciferase transcripts were treated with PAP (18), we decided to test the activity of PAP against uncapped viral RNAs from poliovirus, TBSV and SPMV. Luciferase transcripts lack secondary motifs present in other mRNAs, viral RNAs, and viroids. Poliovirus has an uncapped RNA that contains a secondary structure near the 5' end termed an internal ribosome entry site (IRES) element, which recruits the $40 \mathrm{~S}$ subunits during translation (30). The effect of PAP on transcripts containing poliovirus IRES was tested using a dicistronic mRNA construct, pcDNA3, which contains the luciferase open reading frame followed by an internal chloramphenicol acetyl transferase open reading frame downstream of the poliovirus IRES. In vitro transcripts from the pcDNA3 construct synthesized using T7 RNA polymerase and the in vitro transcripts from plasmids containing the full-length infectious clones of TBSV and SPMV RNAs were incubated with PAP, phenol extracted, and treated with acidic aniline as described previously (18). As shown in Figure 6, depurination by PAP at multiple sites was not observed in the presence of aniline with any one of these RNAs. Although a slight decrease was observed in TBSV RNA after aniline treatment, RNA remained intact and did not show any evidence of smaller cleavage products.

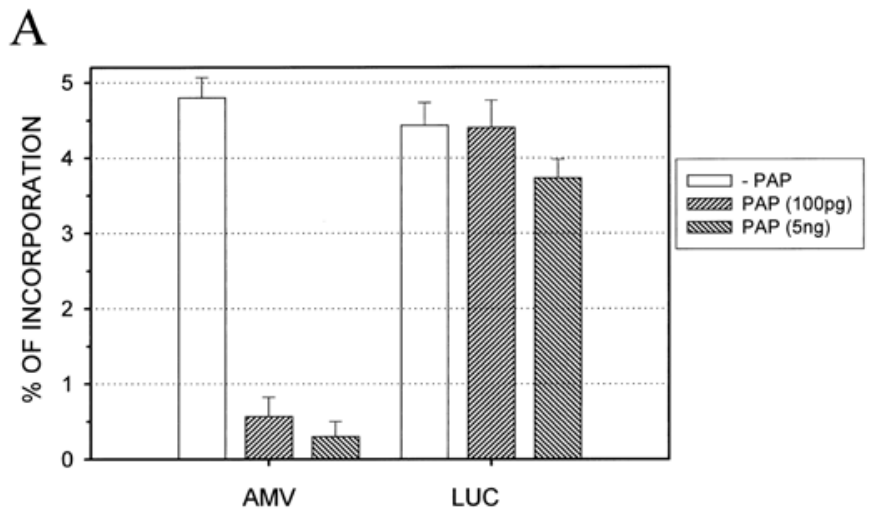

B

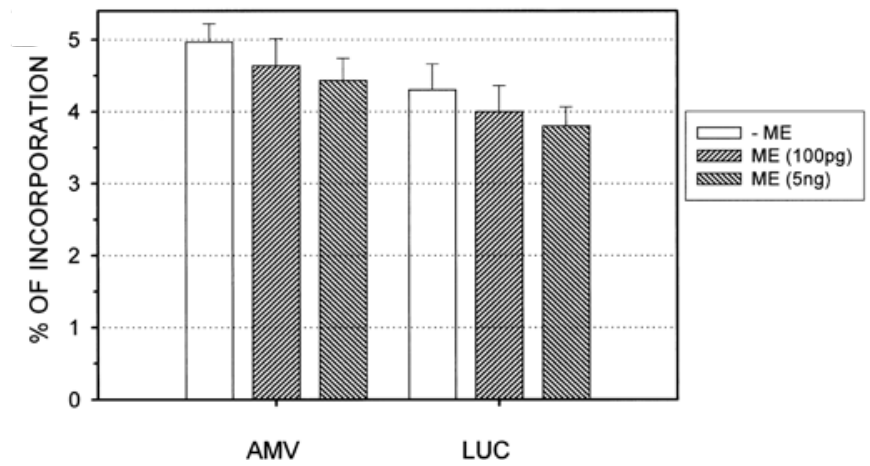

Fig. 5. In vitro translation of Alfalfa mosaic virus (AMV) RNAs and uncapped luciferase (LUC) transcripts treated with pokeweed antiviral protein (PAP) and ME1 from Mirabilis expansa (ME). A, AMV capped RNAs $(500 \mathrm{ng})$ and uncapped luciferase (LUC) transcripts were incubated with $100 \mathrm{pg}$ and $5 \mathrm{ng}$ of PAP as described in the text. PAP was subsequently removed by phenol/chloroform extraction from the incubation mixture, and the ribosome-inactivating protein-treated RNAs were translated in a rabbit reticulocyte lysate system. Percent incorporation indicates the amount of $\left.{ }^{35} \mathrm{~S}\right]$-methionine incorporated into protein compared with the total amount in the translation mixture quantified with a scintillation counter and using untreated RNA as 100\%. B, Capped AMV RNAs (500 ng) and uncapped luciferase (LUC) transcripts were incubated with $100 \mathrm{pg}$ or $5 \mathrm{ng}$ of ME. In vitro translation and analysis were performed as described in A. -PAP and -ME refer to RNA controls incubated with buffer.
To examine translation, transcripts containing polio IRES, and transcripts from full-length infectious clones of TBSV and SPMV were treated with PAP, or buffer without PAP, and translated in rabbit reticulocyte lysate as previously described. As shown in Figure 7A, translation inhibition was observed with all three RNAs treated with $5 \mathrm{ng}$ of PAP (5 ng) compared with translation of the same RNAs incubated with buffer alone (-RIP). To verify the integrity of the PAP-treated RNAs prior to translation, RNAs incubated with PAP were separated on a $7 \mathrm{M}$ urea/ $6 \%$ acrylamide gel. As shown in Figure 7B, uncapped transcripts containing polio IRES and uncapped transcripts from TBSV and SPMV treated with PAP or with buffer were intact, confirming that PAP caused inhibition of translation of these RNAs without detectable RNA depurination. These results demonstrate that PAP can inhibit translation of uncapped viral RNAs without causing detectable depurination at multiple sites and provide further evidence that the cap structure is not the only determinant for inhibition of translation by PAP.

\section{DISCUSSION}

We reported that PAP inhibits translation of capped viral RNAs and that its effect is overcome by increasing concentrations of the cap analog $\mathrm{m}^{7} \mathrm{GpppG}$ (18). When capped and uncapped RNAs were treated with PAP or PAP mutants that could not depurinate ribosomes and assayed for translational competence in cell extracts, translation of capped mRNAs was significantly inhibited. In contrast, PAP had no significant inhibitory effect on uncapped mRNAs (16). These results demonstrate that PAP could inhibit translation by targeting the mRNA rather than the rRNA (19). Analysis of the interaction between PAP and the capped luciferase mRNA shows that PAP binds to the cap structure on mRNA (16). PAP does not depurinate the cap or decap the mRNA; it depurinates the mRNA downstream of the cap at multiple sites throughout its sequence (16). In this article, we describe how PAP and other RIPs, such as ME1 and saporin, inhibit the translation of different capped viral RNAs. ME1 and saporin depurinated the capped BMV RNAs at multiple sites, but not the uncapped luciferase RNA, as previously observed with PAP (18). These results indicate that recognition of cap structure is not unique to PAP, but that other single chain RIPs can distinguish between

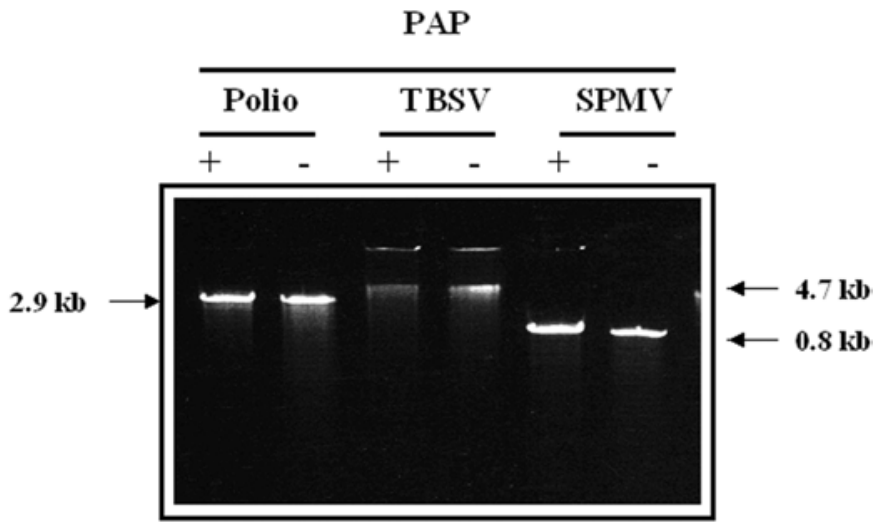

Fig. 6. In vitro incubation of uncapped transcripts containing poliovirus (Polio) IRES and transcripts from Tomato bushy stunt virus (TBSV) and Satellite panicum mosaic virus (SPMV) with pokeweed antiviral protein (PAP). In vitro transcripts containing poliovirus (Polio) internal ribosome entry site (IRES), and transcripts from full-length infectious clones of TBSV and SPMV (500 ng) were incubated with $5 \mathrm{ng}$ of PAP. RNAs were incubated with PAP as described in the text. RNAs were extracted, treated with aniline, separated on a $7 \mathrm{M}$ urea/6\% polyacrylamide gel, and stained with ethidium bromide. The presence $(+)$ or absence $(-)$ of aniline is denoted. Left arrow indicates the $2.9-\mathrm{kb}$ transcript from the pcDNA3 vector containing poliovirus IRES, and right arrows indicate the 4.7-kb TBSV and 0.8-kb SPMV transcripts. 
capped and uncapped RNAs. Capped TMV RNAs showed evidence of depurination only when incubated with higher concentrations of PAP, ME1, and saporin, indicating that the activity of all RIPs was lower against TMV RNA than the activity observed against BMV RNAs. Although TMV and BMV have capped RNAs, the N-glycosidase activity of RIPs differs against the two viral RNAs. These results suggest that the cap structure is important for RIP recognition of the RNA template, but it is not sufficient. Sequences or secondary structures downstream of the $5^{\prime}$ end or at the $3^{\prime}$ ends of these RNAs may play significant roles in RIP recognition and subsequent RNA depurination. BMV and TMV RNAs possess tRNA-like structures at their $3^{\prime}$ ends. It has been reported that tRNAs are involved in the up-regulation of cofactor-requiring RIPs $(6,7)$ and in viral infectivity (41). Thus the presence of tRNA-like structures may have influenced the RIP activity on BMV and TMV RNAs (41).

We tested the depurination activity of RIPs against AMV, a multi-partite capped RNA-containing virus, which does not contain a tRNA-like structure at its $3^{\prime}$ ends. A defining feature of AMV is that genomic RNAs alone are not infectious. Infection is dependent upon combination of genomic RNA and coat protein to initiate replication $(5,37)$. All RIPs tested failed to depurinate AMV RNAs at multiple sites throughout their sequence even at higher concentrations. Only $100 \mathrm{ng}$ of saporin produced detectable depurination of AMV RNAs. Saporin has been shown to depurinate a variety of DNAs, RNAs, and poly(A) (4). Although AMV RNAs are capped, they can be translated in a cap independent manner (12). It is possible that the cap independent translation ability of the AMV RNAs and/or the lack of the tRNA-like structure at their $3^{\prime}$ ends may have accounted for the inability of RIPs to depurinate these RNAs. Although AMV RNAs appeared intact after PAP incubation, PAP inhibited translation of all AMV proteins, indicating that depurination may have occurred in all four RNAs but was not detected by electrophoresis on urea/polyacrylamide gels. Extensive hairpin structures are found near the 5' ends of most AMV RNAs (14). Furthermore, sequences at the 5' ends of AMV RNAs resemble the S/R loop sequences in the context of GAGA. The underlined adenine is the depurination site of RIPs in the S/R loop (10). PAP might have depurinated all four AMV RNAs in sequences resembling the S/R loop. If depurination occurred close to the ends of the RNAs, the depurinated fragments may not be detectable by electrophoresis due to their small size or may have run out of the gel. Under normal circumstances, cap-binding proteins recognize the cap structure allowing the $40 \mathrm{~S}$ ribosomal subunit to bind the mRNA (34). If PAP depurinates the $5^{\prime}$ end of AMV RNAs in such a way that the secondary structure of the RNAs is disrupted, the interaction between the cap structure and the eIF4F complex may be affected. Disruption of the hairpins at the $5^{\prime}$ ends of AMV RNAs may create improper cap accessibility of the cap-binding protein complex (eIF4F), leading to protein synthesis inhibition $(11,14)$. Alternatively, PAP may bind to AMV RNAs and disrupt their translation without depurinating the RNAs. Although neither PAP nor ME1 caused detectable depurination of the AMV RNAs at multiple sites, PAP inhibited translation of these RNAs, whereas ME1 did not have a major inhibitory effect. These results indicate that RIPs differ in their specificity against different capped viral RNAs.

We have previously shown that PAP did not inhibit the translation of uncapped luciferase transcripts, suggesting that PAP did not recognize uncapped RNA templates (18). Based on these results we hypothesized that PAP must recognize the cap structure in order to depurinate the RNA templates. To examine the enzymatic activity of PAP on uncapped viral RNAs, we incubated PAP with full-length infectious transcripts from TBSV and SPMV and transcripts containing the poliovirus IRES element. As expected, PAP did not cause depurination at multiple sites throughout the sequence of these RNAs. However, in vitro translation of all three
RNAs was inhibited after pretreatment with PAP. Similar results were obtained using uncapped Satellite tobacco necrosis virus (STNV) RNA treated with PAP (data not shown). To rule out any RNA degradation due to contaminating nucleases, we showed that the viral RNAs were intact prior to translation. These results indicate that PAP can inhibit translation of uncapped viral RNAs without causing detectable depurination of the RNA at multiple sites. These results are consistent with the previously reported antiviral activity of PAP against viruses that lack $5^{\prime}$ cap structures, such as Potato virus $Y(9,23)$ and poliovirus (40).

Infection of cells with poliovirus, a cytoplasmic RNA virus, results in the modification of several translation initiation factors, such as eIF4F, that are implicated in the efficient recruitment of 40S subunits to capped RNAs (15). Infection by poliovirus results in cleavage of mammalian eIF4G, the large subunit of eIF4F, removing the N-terminally located cap-binding protein (eIF4E) binding site and cap-dependent translation (30). Under these conditions, the translation of polioviral RNA is not inhibited. Poliovirus RNA contains a 450-nucleotide sequence with extensive secondary structure in the viral 5'-noncoding region, termed IRES, which recruits the $40 \mathrm{~S}$ ribosomal subunits (30). For some animal picornaviruses, eIF4G binds to the IRES and promotes internal
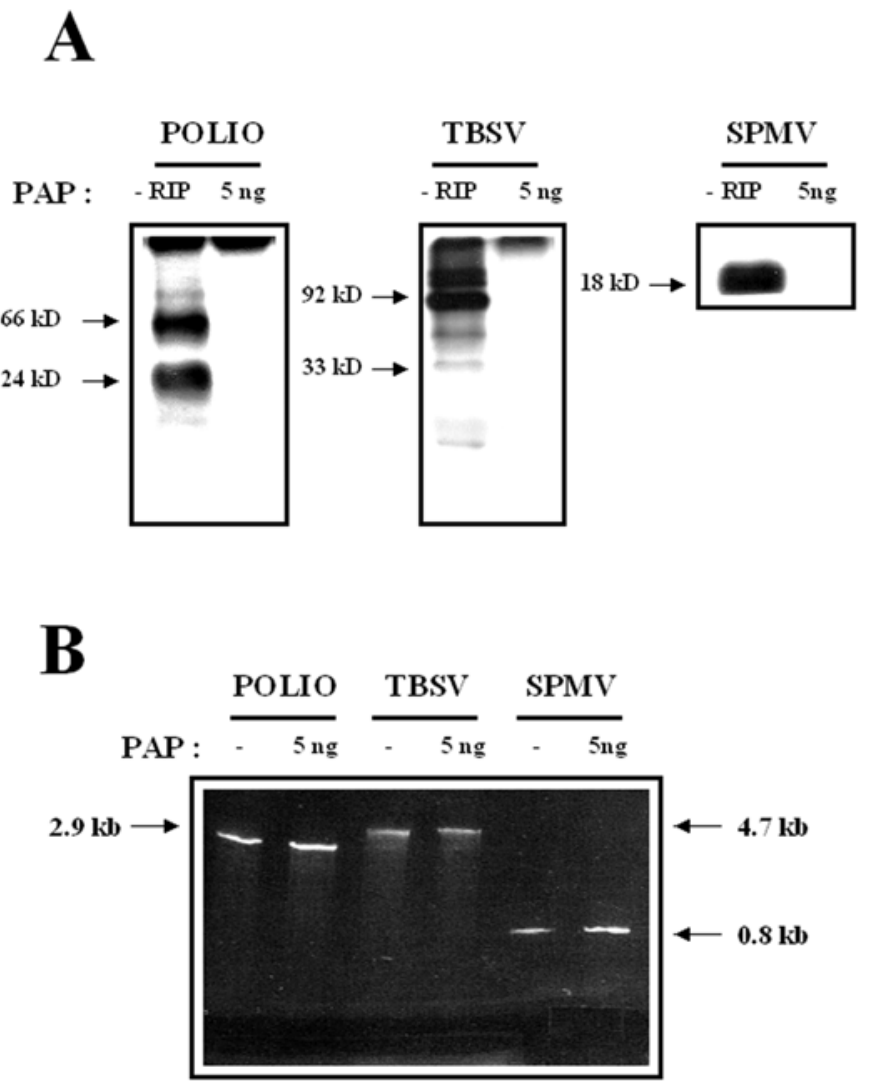

Fig. 7. Autoradiogram illustrating the translation inhibition of uncapped transcripts containing poliovirus (Polio) IRES and transcripts from Tomato bushy stunt virus (TBSV) and Satellite panicum mosaic virus (SPMV) RNAs by pokeweed antiviral protein (PAP), without detectable RNA depurination. A, Viral transcripts (500 ng) were incubated with $5 \mathrm{ng}$ of PAP as described in the text. PAP was subsequently removed by phenol/chloroform extraction from the incubation mixture, and the ribosome-inactivating protein (RIP)treated RNAs were translated in a rabbit reticulocyte lysate translation system. Five microliters of the total $25-\mu \mathrm{l}$ incubation mixture was separated on a $7 \mathrm{M}$ urea/6\% polyacrylamide gel and analyzed by autoradiography. Arrows indicate the proteins translated by the viral RNAs. In Polio lane, the arrows indicate the proteins translated from the pcDNA3 construct, luciferase $(66 \mathrm{kD})$ and CAT $(24 \mathrm{kD})$. B, Viral transcripts were treated with $5 \mathrm{ng}$ of PAP. RNAs were incubated with PAP, extracted, separated on a $7 \mathrm{M}$ urea/ $6 \%$ polyacrylamide gel, and stained with ethidium bromide. RNA controls $(-)$ incubated with buffer are denoted. 
binding of the $40 \mathrm{~S}$ ribosomal subunits (30). Depurination of the IRES element may inhibit its interaction with initiation factors such as eIF4G, resulting in inhibition of its translation. Recent structural analysis of the Hepatitis $C$ virus IRES element showed a motif resembling the S/R loop of the large rRNA (19). Similarly, TSBV and SPMV RNAs may have secondary structures at their $5^{\prime}$ or $3^{\prime}$ ends $(25,42)$ that may be sensitive to PAP-mediated depurination. Further studies will address if the uncapped RNAs are depurinated by PAP.

The biological role of RIPs in plants has not yet been elucidated. An antiviral role has been proposed for some RIPs such as PAP. Based on their enzymatic activity and cellular location, it has been suggested that RIPs may be suicidal agents released from cell walls or vacuoles after a plant cell is damaged by viral infection. Consistent with this view, the induction of RIP activity has been observed in virus-infected sugar beet plants (13). We have shown that the relative affinity of PAP for the S/R loop of the rRNA is only fourfold greater than its affinity for capped RNA (16). Given that cellular RNA is comprised of $85 \%$ rRNA and only a few percent mRNA, PAP may preferentially depurinate ribosomes. However, during viral infection, when the concentration of PAP or the viral RNA increases, viral RNA may become a substrate for PAP (16). We recently showed that PAP affects the stability of its own mRNA in vivo, providing evidence that PAP can target an mRNA, in addition to the rRNA in vivo (29). Viruses such as Pokeweed mosaic virus may infect pokeweed plants due to structural modifications, including the absence of a $5^{\prime}$ cap and a 3' tRNA-like structure, which may enable them to withstand the inhibitory effects of PAP in pokeweed. In the last decade, some RIPs have gained widespread interest because of their use as immunotoxins directed against malignant cells. Our results may increase the potential of RIPs as therapeutic agents by targeting their activity to specific viral pathogens.

\section{ACKNOWLEDGMENTS}

This work was supported by an NSF grant MCB 9982498 to N. Tumer. We thank H. Scholthof and K.-B. Scholthof for plasmids containing fulllength infectious clones of TBSV and SPMV RNAs and purified virus preparations, N. Sonenberg from pcDNA3 containing poliovirus IRES sequences, and K. Browning for cDNA clone of STNV used in this work.

\section{LITERATURE CITED}

1. Baer, M., Houser, F., Loesch-Fries, L. S., and Gehrke, L. 1994. Specific RNA binding by amino-terminal peptides of alfalfa mosaic virus coat protein. EMBO J. 13:727-735.

2. Barbieri, L., Gorini, P., Valbonesi, P., Castiglioni, P., and Stirpe, F. 1994. Unexpected activity of saporins. Nature 372:624.

3. Barbieri, L., Valbonesi, P., Bonora, E., Gorini, P., Bolognesi, A., and Stirpe, F. 1997. Polynucleotide: adenosine glycosidase activity of ribosome-inactivating proteins: Effect on DNA, RNA and poly (A). Nucleic Acids Res. 25:518-522.

4. Barbieri, L., Valbonesi, P., Gorini, P., Pession, A., and Stirpe, F. 1996. Polynucleotide: adenosine glycosidase activity of saporin-L1: Effect on DNA, RNA and poly (A). Biochem. J. 319:507-513.

5. Bol, J. F., van Vloten-Doting, L., and Jaspars, E. M. J. 1971. A functional equivalence of top component $a$ RNA and coat protein in the initiation of infection by alfalfa mosaic virus. Virology 46:73-85.

6. Brigotti, M., Carnicelli, D., Alvergna, P., Pallanca, A., Sperti, S., and Montanaro, L. 1995. Differential up-regulation by tRNAs of ribosomeinactivating proteins. FEBS Lett. 373:115-118.

7. Brigotti, M., Keith, G., Pallanca, A., Carnicelli, D., Alvergna, P., Dirheimer, G., Montanaro, L., and Sperti, S. 1998. Identification of the tRNAs which up-regulate agrostin, barley RIP and PAPs, three ribosome inactivating proteins of plant origin. FEBS Lett. 431:259-262.

8. Carzaniga, R., Sinclair, L., Fordham-Skelton, A. P., Harris, N., and Croy, R. R. D. 1994. Cellular and subcellular distribution of saporins, type-1 ribosome-inactivating proteins, in soapwort (Saponaria officinalis L.). Planta 194:461-470.

9. Chen, Z., White, R. F., Antoniw, J. F., and Lin, Q. 1991. Effect of pokeweed antiviral protein (PAP) on the infection of plant viruses. Plant Pathol. 40:612-620.
10. Endo, Y., Mitsui, K., Motizuki, M., and Tsurugi, K. 1987. The mechanism of action of ricin and related toxic lectins on eukaryotic ribosomes. J. Biol. Chem. 262:5908-5912.

11. Fletcher, L., Corbin, S. D., Browning, K. S., and Ravel, J. M. 1990. The absence of a m7G cap on $\beta$-Globin mRNA and Alfalfa Mosaic Virus RNA 4 increases the amounts of initiation factor $4 \mathrm{~F}$ required for translation. J. Biol. Chem. 265:19582-19587.

12. Gehrke, L., Auron, P. E., Quigley, G. J., Rich, A., and Sonenberg, N. 1983. 5'-Conformation of capped alfalfa mosaic virus ribonucleic acid 4 may reflect its independence of the cap structure or of cap-binding protein for efficient translation. Biochemistry 22:5157-5164.

13. Girbes, T., de Torre, C., Iglesias, R., Ferreras, J. M., and Mendez, E. 1996. RIP for viruses. Nature 379:777-778.

14. Godefroy-Colburn, T., Ravelonandro, M., and Pinck, L. 1985. Cap accessibility correlates with the initiation efficiency of alfalfa mosaic virus RNAs. Eur. J. Biochem. 147:549-552.

15. Gradi, A., Imataka, H., Svitkin, Y. V., Rom, E., Raught, B., Morino, S., and Sonenberg, N. 1998. A novel functional human eukaryotic translation initiation factor 4G. Mol. Cell. Biol. 18:334-342.

16. Hudak, K. A., Bauman, J. D., and Tumer, N. E. 2002. Pokeweed antiviral protein binds to the cap structure of eukaryotic mRNA and depurinates the mRNA downstream of the cap. RNA 8:1148-1159.

17. Hudak, K. A., Dinman, J. D., and Tumer, N. E. 1999. Pokeweed antiviral protein accesses ribosomes by binding to L3. J. Biol. Chem. 274:38593864.

18. Hudak, K. A., Wang, P., and Tumer, N. E. 2000. A novel mechanism for inhibition of translation by pokeweed antiviral protein: Depurination of the capped RNA template. RNA 6:369-380.

19. Klinck, R., Westhof, E., Walker, S., Afshar, M., Collier, A., and AboulEla, F. 2000. A potential RNA drug target in the hepatitis C virus internal ribosomal entry site. RNA 6:1423-1431.

20. Laemmli, U. K. 1970. Cleavage of structural proteins during the assembly of the head of bacteriophage T4. Nature 227:680-685.

21. Li, M. X., Yeung, H. W., Pan, L. P. and Chan, S. I. 1991. Trichosanthin, a potent HIV-1 inhibitor, can cleave supercoiled DNA in vitro. Nucleic Acids Res. 19:6309-6312.

22. Ling, J., Liu, W., and Wang, T. P. 1994. Cleavage of supercoiled doublestranded DNA by several ribosome-inactivating proteins in vitro. FEBS Lett. 345:143-146.

23. Lodge, J. K., Kaniewski, W. K., and Tumer, N. E. 1993. Broad-spectrum virus resistance in transgenic plants expressing pokeweed antiviral protein. Proc. Natl. Acad. Sci. USA 90:7089-7093.

24. Montanaro, L., Sperti, S., Mattioli, A., Testoni, G., and Stirpe, F. 1975. Inhibition by ricin of protein synthesis in vitro. Inhibition of the binding of elongation factor 2 and of adenosine diphosphate-ribosylated elongation factor 2 to ribosomes. Biochem. J. 146:127-131.

25. Morris, T. J., and Knorr, D. A. 1990. Defective interfering viruses associated with plant virus infections. Pages 123-127 in: New Aspects of Positive Strand RNA Viruses. M. A. Brinton and F. X. Heinz, eds. American Society of Microbiology, Washington, D.C.

26. Nicolas, E., Beggs, J. M., Haltiwanger, B. M., and Taraschi, T. F. 1998. A new class of DNA glycosylase/apurinic/apyrimidinic lyases that act on specific adenines in single-stranded DNA. J. Biol. Chem. 273:1721617220.

27. Nielsen, K., and Boston, R. S. 2001. Ribosome-inactivating proteins: A plant perspective. Annu. Rev. Plant Physiol. Plant Mol. Biol. 52:785816.

28. Osborn, R. W., and Hartley, M. R. 1990. Dual effects of the ricin Achain on protein synthesis in rabbit reticulocyte lysate. Inhibition of initiation and translocation. Eur. J. Biochem. 193:401-417.

29. Parikh, B. A., Coetzer, C., and Tumer, N. E. 2002. Pokeweed antiviral protein regulates the stability of its own mRNA by a mechanism that requires depurination but can be separated from depurination of the $\alpha$ sarcin/ricin loop of rRNA. J. Biol. Chem. 277:41428-41437.

30. Pestova, T. V., Kolupaeva, V. G., Lomakin, I. V., Pilipenko, E. V., Shatsky, I. N., Agol, V. I., and Hellen, C. U. T. 2001. Molecular mechanisms of translation initiation in eukaryotes. Proc. Natl. Acad. Sci USA 98:7029-7036.

31. Ready, M., Brown, D. T., and Robertus, J. D. 1986. Extracellular localization of pokeweed antiviral protein. Proc. Natl. Acad. Sci. USA 83:5053-5056.

32. Roberts, L. M., and Lord, J. M. 1981. The synthesis of Ricinus communis agglutinin-cotranslational and posttranslational modification of agglutinin polypeptides. J. Eur. Biochem. 119:31-41.

33. Roncuzzi, L., and Gasperi-Campani, A. 1996. DNA-nuclease activity of single-chain ribosome-inactivating proteins dianthin 30, saporin 6 and gelonin. FEBS Lett. 392:16-20.

34. Sonenberg, N., Trachsel, H., Hecht, S., and Shatkin, A. J. 1980. Differential stimulation of capped mRNA translation in vitro by cap binding 
protein. Nature 285:331-333.

35. Swanson, M. M., Ansel-Mckinney, P., Houser-Scott, F., Vidadi, Y., Loesch-Fries, L. S., and Gehrke, L. 1998. Viral coat protein peptides with limited sequence homology bind similar domains of Alfalfa mosaic virus and tobacco streak virus RNAs. Virology 72:3227-3234.

36. Tumer, N. E., Hwang, D. J., and Bonness, M. 1997. C-terminal deletion mutant of pokeweed antiviral protein inhibits viral infection but does not depurinate host ribosomes. Proc. Natl. Acad. Sci. USA 94:3866-3871.

37. van Vloten-Doting, L. 1975. Coat protein is required for infectivity of tobacco streak virus: Biological equivalence of the coat proteins of tobacco streak and alfalfa mosaic virus. Virology 65:215-225.

38. Vivanco, J. M., Savary, B. J., and Flores, H. E. 1999. Characterization of two novel type I ribosome-inactivating proteins from the storage roots of the Andean crop Mirabilis expansa (Ruiz \& Pavon). Plant Physiol. 119:1447-1456.
39. Wang, P., and Tumer, N. E. 1999. Pokeweed antiviral protein cleaves double-stranded supercoiled DNA using the same active site required to depurinate rRNA. Nucleic Acids Res. 27:1900-1905.

40. Wang, P., and Tumer, N. E. 2000. Virus resistance mediated by ribosome inactivating proteins. Adv. Virus Res. 55:325-356.

41. Weiner, A. M., and Maizels, N. 1987. tRNA-like structures tag the $3^{\prime}$ ends of genomic RNA molecules for replication: Implications for the origin of protein synthesis. Proc. Natl. Acad. Sci. USA 84:7383-7387.

42. Wenping, Q., and Scholthof, K.-B. G. 2000. In vitro- and in vivogenerated defective RNAs of satellite panicum mosaic virus define cisacting RNA elements required for replication and movement. J. Virol. $74: 2247-2254$

43. Zoubenko, O., Uckun, F., Hur, Y., Chet, I., and Tumer, N. E. 1997. Plant resistance to fungal infection by nontoxic pokeweed antiviral protein mutants. Nat. Biotech. 15:992-996. 